University of Nebraska - Lincoln

DigitalCommons@University of Nebraska - Lincoln

US Army Research

U.S. Department of Defense

2011

\title{
A new explanation of the extinction paradox
}

Matthew J. Berg

US Army Research Laboratory, Adelphi, MD, matt.berg@msstate.edu

C. M. Sorensen

Kansas State University

A. Chakrabarti

Kansas State University

Follow this and additional works at: https://digitalcommons.unl.edu/usarmyresearch

Part of the Operations Research, Systems Engineering and Industrial Engineering Commons

Berg, Matthew J.; Sorensen, C. M.; and Chakrabarti, A., "A new explanation of the extinction paradox" (2011). US Army Research. 110.

https://digitalcommons.unl.edu/usarmyresearch/110

This Article is brought to you for free and open access by the U.S. Department of Defense at DigitalCommons@University of Nebraska - Lincoln. It has been accepted for inclusion in US Army Research by an authorized administrator of DigitalCommons@University of Nebraska - Lincoln. 


\title{
A new explanation of the extinction paradox
}

\author{
M.J. Berg ${ }^{\text {a,b,* }}$, C.M. Sorensen ${ }^{c}$, A. Chakrabarti ${ }^{c}$ \\ ${ }^{a}$ Mississippi State University, Department of Physics E Astronomy, Mississippi State, MS 39762, USA \\ ${ }^{\mathrm{b}}$ US Army Research Laboratory, RDRL-CIE-S, 2800 Powder Mill Road, Adelphi, MD 20783, USA \\ ${ }^{\mathrm{c}}$ Kansas State University, Department of Physics, Manhattan, KS 66506, USA
}

\section{A R T I C L E I N F O}

\section{Available online 9 September 2010}

Keywords:

Extinction paradox

Extinction

Ewald-Oseen

Geometrical optics

Light scattering

\begin{abstract}
A B S T R A C T
This work presents a new explanation for the extinction paradox and shows that the canonical explanations are incorrect. This paradox refers to the large size limit of a particle's extinction cross section. It is called a paradox because the geometrical optics approximation, which should be valid in this limit, predicts a cross section that is half of the true value. The new explanation is achieved by formulating the scattered wave in terms of an integral over the particle's surface where the seemingly unrelated Ewald-Oseen theorem appears in the formulation. By expressing the cross section in terms of this surface integral, the Ewald-Oseen theorem is analytically connected to the cross section. Several illustrations are used to reveal the significance of this connection: The paradox is seen to be a consequence of the requirement that the incident wave be canceled within the particle by secondary radiation from its own internal field. Following this, the canonical explanations are examined to reveal serious problems. In the process, the same asymptotic extinction behavior is shown to occur for small highly refractive dielectric particles, and thus is not just a large particle size or small wavelength effect as is often stated. The traditional explanations cannot account for this behavior while the new one actually predicts it. All in all, this work constitutes a fundamental reworking of 60 years of accepted understanding for the cause of the asymptotic behavior of the extinction cross section.
\end{abstract}

(c) 2010 Elsevier Ltd. All rights reserved.

\section{Introduction}

The extinction paradox traditionally refers to the asymptotic limit of a particle's extinction cross section as the size of the particle becomes much larger than the incident wavelength [1,2]. The reason this is called a paradox is due to an attempt to understand extinction using the geometrical optics approximation, which should be valid for such very large particles. In this approximation, the cross section $C^{\text {ext }}$ is expected to equal the particle's geometrical cross section $C^{\text {geo }}$. However, the true value as properly measured or calculated from exact

\footnotetext{
* Corresponding author at: Mississippi State University, Department of Physics \& Astronomy, Mississippi State, MS 39762, USA.

E-mail address: matt.berg@msstate.edu (M.J. Berg).
}

theory is twice this, i.e., $C^{\text {ext }}=2 C^{\text {geo }}$. One might then ask, how can a very large particle remove more energy from a beam than it geometrically intercepts? Explanations for this discrepancy are well established. The most often cited is due to van de Hulst and relies on a combination of diffraction and geometrical optics [3]. A less encountered alternative, by Brillouin, relies on destructive interference within the geometrical shadow [4]. The highly intuitive and simple character of these enduring explanations has given the paradox a reputation as a well-understood effect.

The purpose of this work is twofold: A new analytical framework will be presented to describe the asymptotic extinction behavior associated with the paradox. This is done by deriving a connection between the cross section and the seemingly unrelated Ewald-Oseen (EO) theorem. Numerical examples are presented in Figs. 3-6, which 
develop for the reader an intuition for the role of the EO theorem in the scattering process. When taken together, these examples reveal a connection between EO and the paradox. It is this connection that leads to a new explanation of the paradox and uncovers important insight into the fundamental wave nature of extinction. The second purpose of this paper is to demonstrate that the canonical explanations of the paradox fail. In particular, one will see that they cannot explain why the same asymptotic extinction behavior occurs for a wavelength-sized particle that is highly refractive, or why it occurs in both the near and far-field zones of any particle. The new explanation addresses these cases and provides a simple account for why the paradox occurs in general.

\section{The flower pot}

The common explanation for the paradox is based on a combination of diffraction and geometrical optics [3]: The particle is assumed to be much larger than the incident wavelength. The incident wave is then viewed as an infinitely wide beam of parallel rays traveling along the forward direction, which are separated in two groups; those that intercept the particle's geometrically illuminated surface and those that do not. The separation between these groups occurs at the particle's shadow boundary, which is a contour separating the illuminated and shaded sides. The intercepted rays are either reflected, refracted, or absorbed and occupy a transverse area of the incident wavefront equal to $C^{\text {geo }}$. The remaining rays, i.e., those not intercepted by the particle, represent an incomplete wavefront at the shadow boundary with an area of $C^{\text {geo }}$ absent. This wavefront then diffracts just as a complete wavefront would in passing by an opaque disk-like obstacle with the same shape and size as $C^{\text {geo }}$.

A tacit assumption here is that the meaning of $C^{\text {ext }}$ corresponds to the removal of energy flow from the forward direction. If so, then the intercepted and incomplete portions of the wavefront each contribute a factor of $C^{\text {geo }}$ to $C^{\text {ext }}$, for a total of $2 C^{\text {geo }}$. Hence, the paradox is apparently explained. Examples of work that promote this diffraction-based explanation are numerous, some of which in electromagnetic theory can be found in [1-3,5-13], while the quantum mechanical analog is discussed in [14].

In illustrating his diffraction-based explanation, van de Hulst describes a flower pot that is placed in a window [3, p. 107]. He states that, “... [the pot] prevents only the sunlight falling on it from entering the room, and not twice this amount, but a meteorite of the same size somewhere in interstellar space between a star and one of our big telescopes will screen twice this light." Thus, if $C^{\text {ext }}$ represents the loss of light entering the room, then one should not find the paradoxical value of $2 C^{\text {geo }}$. Consequently, van de Hulst adds the requirement that "...the observation [of $C^{\text {ext }}$ ] is made at very great distance, i.e., far beyond the zone where a shadow can be distinguished." In other words, far-field diffraction from the pot's shadow boundary needs enough distance over which to deflect light out of the forward direction before $C^{\text {ext }}=2 C^{\text {geo }}$ is expected.

This explanation is simple and intuitive, but not without substantial problems. For example, it is possible to prove analytically, directly from the Maxwell equations, that the value of $C^{\text {ext }}$ for any arbitrary particle cannot depend on how far from the particle it is calculated, see [15]. This means that one must find that $C^{\text {ext }}=2 C^{\text {geo }}$ not only in the pots's far-field zone, but also in the near-field zone, even infinitesimally close to the pot's surface. Other problems are discussed in Section 5.1.

The new explanation is free from these problems. In short, it works by realizing that there is a connection between the EO theorem and the mathematical expression yielding $C^{\text {ext }}$. This requires that any particle remove the incident wave from its interior via secondary radiation from its own internal polarization wave. This secondary radiation cancels the incident wave through destructive interference. The source derived from the internal wave producing this secondary radiation is the same source that produces the external scattered wave. Thus, the requirement that the incident wave be canceled is communicated outside the particle via its scattered wave, and hence also to $C^{\text {ext }}$. Although further detail must wait until later, it is possible now to see how $C^{\text {ext }}=2 C^{\text {geo }}$ for the flower pot by making simple observations of the energy flow in the pot's near-field zone. In doing so, an effort will be made to involve the concepts of geometrical optics as much as possible since these concepts are expected to be valid for this example.

To begin, suppose that the pot can be treated like a perfect conductor. Then, if it is possible to account for the pot's scattering cross section $C^{\text {sca }}, C^{\text {ext }}$ will follow from the conservation of energy, Eq. (10) below. This is because a perfect conductor's absorption cross section is $C^{\text {abs }}=0$, hence $C^{\text {ext }}=C^{\text {sca }}$. First, notice that there is a well-defined dark shadow immediately behind the pot. Then, the amount of power contained in the portion of the scattered wave that occupies this geometrical shadow must be equal to that of the incident wave. This is because the scattered wave must cancel the incident wave there through destructive interference. Therefore, the observation of a well-defined shadow means a contribution of $C^{\text {geo }}$ to $C^{\text {sca }}$. An additional factor of $C^{\text {geo }}$ is supplied by reflection of the light geometrically incident upon the pot's illuminated side as required by energy conservation. Thus, the total scattering cross section is $C^{\text {sca }}=2 C^{\text {geo }}$ giving $C^{\text {ext }}=2 C^{\text {geo }}$ as expected. Sections 4.4 and 4.5 will show that the shadow forming part of the scattered wave is actually the EO cancellation wave propagating outside of the particle. Moreover, one will see that the double burden of producing both the scattered and EO cancellation waves results in the particle's internal-wave source having twice the magnitude otherwise expected to produce either wave alone. This factor of two and the one in the paradox are one in the same.

Now suppose that the pot is highly absorbing, i.e., not a perfect conductor but is still completely opaque. As before, conservation of energy can be used to find $C^{\text {ext }}$, except now this requires specification of both $C^{\text {sca }}$ and $C^{\text {abs. }}$. If the pot is absorbing enough such that all of the 
light geometrically incident upon it is lost, then $C^{\text {abs }}=C^{\text {geo }}$ and there is a well-defined shadow. One might now be tempted to think that $C^{\text {sca }}=0$ since the pot absorbs all of the light that it intercepts. But, in fact $C^{\text {sca }}=C^{\text {geo }}$ because, again, a well-defined shadow can only happen if there is another wave, the scattered wave, in the shadow region that cancels the incident wave; it is not enough for the scattered wave to just be zero. Then, totaling the contributions, $C^{\text {abs }}=C^{\text {geo }}$, and $C^{\text {sca }}=C^{\text {geo }}$, which gives $C^{\text {ext }}=2 C^{\text {geo }}$ as expected.

The incident wave must be canceled inside both the perfectly conducting and absorbing pots. Absorption cannot achieve this cancellation. Rather, absorption describes the attenuation of the internal wave. Suppose that this wave is attenuated so strongly that it is essentially zero throughout the pot except in a thin region near its illuminated surface. Thus, the perfectly conducting and absorbing pots are the same in this sense. There is an internal field induced at the surface of either pot, and it is this field that becomes the source responsible for EO cancellation, and concomitantly the external scattered wave. Thus, the formation of the welldefined shadow behind these pots is really a consequence of EO cancellation within either pot's interior. This is the crucial recognition missed in the traditional explanations since $C^{\text {ext }}$ is not formulated in terms of its source in the particle's internal field. Moreover, this shows that even a glass flower pot will have $C^{\text {ext }}=2 C^{\text {geo }}$ due to the fundamental requirement that its incident wave be canceled within. One will see this explicitly in Sections 4.1-4.3. Notice that there is no need to rely on any concept of diffraction from the pot's shadow boundary, and no need to observe the pot in the far-field zone in order to explain the paradox.

In the following, Section 3 will review the required mathematical aspects of electromagnetic energy flow, the extinction cross section, and the related efficiency factor. Section 4 develops the connection between EO and $C^{\text {ext }}$ and numerically investigates the connection to illustrate its significance. Figs. 3-7 are particularly important because they collectively demonstrate that the paradox is not limited to large size parameters or opaque particles. The reader not interested in the mathematical justification of this work may skip to Section 4.5 where the primary significance of the calculations is described. Section 5 revisits the canonical explanations and compares them to the EO explanation.

\section{Mathematical preliminaries}

Perhaps the simplest presentation of the paradox is to consider a nonmagnetic spherical particle of radius $R$ and refractive index $m$ residing in vacuum and illuminated by a linearly polarized plane wave. The fields of this wave are

$\mathbf{E}^{\text {inc }}(\mathbf{r})=\mathbf{E}_{\mathrm{o}}^{\text {inc }} \exp \left(i k r \hat{\mathbf{r}} \cdot \hat{\mathbf{n}}^{\text {inc }}\right)$,

$\mathbf{B}^{\text {inc }}(\mathbf{r})=\frac{k}{\omega} \hat{\mathbf{n}}^{\text {inc }} \times \mathbf{E}^{\text {inc }}(\mathbf{r})$.

The vector $\mathbf{E}_{\mathrm{o}}^{\text {inc }}$ describes the amplitude and polarization of this incident wave and $k$ is the wavenumber $2 \pi / \lambda$, where $\lambda$ is the vacuum wavelength. All field quantities in this work are time-harmonic, described by the factor $\exp (-i \omega t)$, where $\omega=k c$, with $c$ being the speed of light. This time factor will be suppressed for brevity. A spherical particle is chosen because Mie theory can be used to calculate the fields and cross sections exactly, following [1]. The essential conclusions of this work are general, however, and apply to any particle shape. Let the surface and interior volume of the particle be $\mathcal{S}$ and $V^{\text {int }}$, respectively. The particle is centered at the origin and enclosed by an imaginary spherical surface $\mathcal{S}^{\text {en }}$ of radius $R_{\text {en }}$ and normal $\hat{\mathbf{n}}^{\text {en }}$, see Fig. 1 . The volume bounded by $\mathcal{S}^{\text {en }}$, excluding $V^{\text {int }}$, the external volume $V^{\text {ext }}$.

The total wave that exists when the particle is present is deliberately decomposed into the superposition of the original incident wave and a modification, called the scattered wave, i.e.,

$\mathbf{E}(\mathbf{r})=\mathbf{E}^{\text {inc }}(\mathbf{r})+\mathbf{E}^{\mathrm{sca}}(\mathbf{r})$

and

$\mathbf{B}(\mathbf{r})=\mathbf{B}^{\text {inc }}(\mathbf{r})+\mathbf{B}^{\mathrm{sca}}(\mathbf{r})$.

This is done so that the effects associated with the introduction of the particle, i.e., those of electromagnetic scattering, can be expressed mathematically in terms of the scattered fields $\mathbf{E}^{\text {sca }}$ and $\mathbf{B}^{\text {sca }}$, see [16].

Typical detectors respond to the time-averaged energy flow of the total wave, which is given in terms of the fields by the Poynting vector $[3,17]$

$$
\langle\mathbf{S}\rangle_{t}=\frac{1}{2 \mu_{\mathrm{o}}} \operatorname{Re}\{\mathbf{E}(\mathbf{r}) \times \mathbf{B}(\mathbf{r})\} .
$$

Combination of Eqs. (3)-(5) shows that the Poynting vector factors into three terms

$\langle\mathbf{S}\rangle_{t}=\left\langle\mathbf{S}^{\text {inc }}\right\rangle_{t}+\left\langle\mathbf{S}^{\text {sca }}\right\rangle_{t}+\left\langle\mathbf{S}^{\text {ext }}\right\rangle_{t}$,

where $\left\langle\mathbf{S}^{\text {inc }}\right\rangle_{t}$ involves only the incident fields, $\left\langle\mathbf{S}^{\text {sca }}\right\rangle_{t}$ involves the scattered fields, and $\left\langle\mathbf{S}^{\text {ext }}\right\rangle_{t}$ involves the

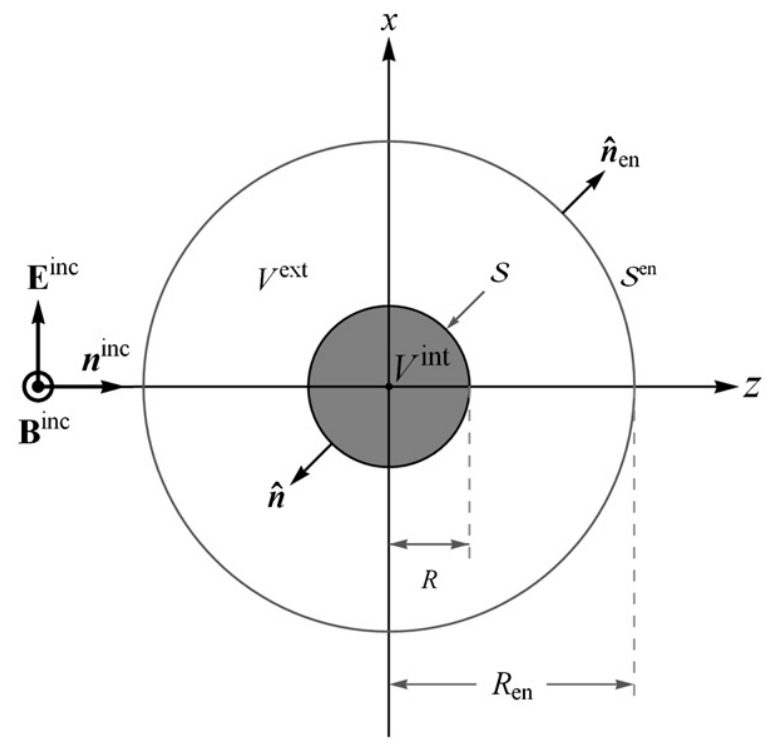

Fig. 1. Arrangement used to derive $C^{\text {ext }}$ and $Q^{\text {ext }}$. 
product of the incident and scattered fields

$$
\begin{aligned}
\left\langle\mathbf{S}^{\text {ext }}\right\rangle_{t}= & \frac{1}{2 \mu_{\mathrm{o}}} \operatorname{Re}\left\{\mathbf{E}^{\text {inc }}(\mathbf{r})\right. \\
& \left.\times\left[\mathbf{B}^{\text {sca }}(\mathbf{r})\right]^{*}+\mathbf{E}^{\text {sca }}(\mathbf{r}) \times\left[\mathbf{B}^{\text {inc }}(\mathbf{r})\right]^{*}\right\} .
\end{aligned}
$$

The extinction cross section $C^{\text {ext }}$ is obtained by integrating the component of Eq. (7) flowing into $\mathcal{S}^{\text {en }}$, giving

$$
\begin{aligned}
C^{\text {ext }}= & -\frac{1}{2 \mu_{\mathrm{o}} I^{\text {inc }}} \operatorname{Re} \oint_{\mathcal{S}_{\text {en }}}\left\{\mathbf{E}^{\text {inc }}(\mathbf{r}) \times\left[\mathbf{B}^{\text {sca }}(\mathbf{r})\right]^{*}\right. \\
& \left.+\mathbf{E}^{\text {sca }}(\mathbf{r}) \times\left[\mathbf{B}^{\text {inc }}(\mathbf{r})\right]^{*}\right\} \cdot \hat{\mathbf{r}} \mathrm{d} S,
\end{aligned}
$$

where $I^{\text {inc }}=(1 / 2) \sqrt{\varepsilon_{0} / \mu_{\mathrm{o}}}\left|\mathbf{E}_{\mathrm{o}}^{\text {inc }}\right|^{2}$. Similarly, the scattering and absorption cross sections $C^{\text {sca }}$ and $C^{\text {abs }}$ are given by the integration of $\left\langle\mathbf{S}^{\text {sca }}\right\rangle_{t}$ and $\langle\mathbf{S}\rangle_{t}$ over $\mathcal{S}^{\text {en }}$, respectively as shown in [17, p. 57].

An especially important quantity will be the extinction efficiency factor

$Q^{\text {ext }}=\frac{C^{\text {ext }}}{C^{\text {geo }}}$

The meaning of $Q^{\text {ext }}$ is the amount of power removed from the region bounded by $\mathcal{S}^{\text {en }}$, due to scattering and absorption, relative to the amount of power contained in the portion of the incident wave geometrically intercepted by the particle [17, p. 59, 1, p. 72]. The paradox will be seen in $Q^{\text {ext }}$ as $Q^{\text {ext }} \rightarrow 2$. More generally, $C^{\text {ext }}$ relates to the conservation of energy as given by [17, p. 57]

$C^{\mathrm{ext}}=C^{\mathrm{sca}}+C^{\mathrm{abs}}$.

In short, $C^{\text {sca }}$ and $C^{\text {abs }}$ represent losses to the energy contained in $\mathcal{S}^{\text {en }}$; the scattered wave carries energy out through $\mathcal{S}^{\text {en }}$, and absorption converts energy into other forms and thus acts as a sink. Consequently, the meaning of extinction refers to the net energy lost through $\mathcal{S}^{\text {en }}$. Notice, that this does not necessarily mean that $C^{\text {ext }}$ represent the reduction of energy flowing along the direction of the incident wave, see [18-20].

\section{Ewald-Oseen and its relation to the paradox}

Eq. (8) yields $C^{\text {ext }}$ in terms of the particle's scattered fields on $\mathcal{S}^{\text {en }}$, and is valid at any distance from the particle. However, the more common approach is to expand $\mathcal{S}^{\text {en }}$ to infinity and use the optical theorem to find $C^{\text {ext }}$ as [17, p. 36]

$C^{\text {ext }}=\frac{4 \pi}{k\left|\mathbf{E}_{\mathrm{o}}^{\mathrm{inc}}\right|^{2}} \operatorname{Im}\left\{\left[\mathbf{E}_{\mathrm{o}}^{\mathrm{inc}}\right]^{*} \cdot \mathbf{E}_{1}^{\mathrm{sca}}\left(\hat{\mathbf{n}}^{\mathrm{inc}}\right)\right\}$.

The function $\mathbf{E}_{1}^{\text {sca }}$ in Eq. (11) is the scattering amplitude, defined by

$\mathbf{E}^{\mathrm{sca}}(\mathbf{r})=\frac{\exp (i k r)}{r} \mathbf{E}_{1}^{\mathrm{sca}}(\hat{\mathbf{r}}), \quad k r \rightarrow \infty$

Using the volume integral equation (VIE), the scattered wave is [17, p. 35]

$$
\mathbf{E}^{\mathrm{sca}}(\mathbf{r})=c_{\mathrm{o}} \int_{V_{\text {int }}} \stackrel{\leftrightarrow}{G}_{\mathrm{e}}\left(\mathbf{r}, \mathbf{r}^{\prime}\right) \cdot \mathbf{E}^{\mathrm{int}}\left(\mathbf{r}^{\prime}\right) \mathrm{d} \mathbf{r}^{\prime},
$$

where $c_{0}=k^{2}\left(m^{2}-1\right)$. In Eq. (13),

$$
\stackrel{\leftrightarrow}{G}_{\mathrm{e}}\left(\mathbf{r}, \mathbf{r}^{\prime}\right)=\left(\overleftrightarrow{I}+\frac{1}{k^{2}} \nabla \otimes \nabla\right) \frac{\exp \left(i k\left|\mathbf{r}-\mathbf{r}^{\prime}\right|\right)}{4 \pi\left|\mathbf{r}-\mathbf{r}^{\prime}\right|},
$$

is the dyadic electric Green's function. There is also a dyadic magnetic Green's function, which will be needed later and is related to $\stackrel{G}{\mathrm{e}}_{\mathrm{e}}$ as

$\stackrel{\leftrightarrow}{G}_{\mathrm{m}}\left(\mathbf{r}, \mathbf{r}^{\prime}\right)=\nabla \times \overleftrightarrow{G}_{\mathrm{e}}\left(\mathbf{r}, \mathbf{r}^{\prime}\right)$

Conceptually, one can envision $\stackrel{\leftrightarrow}{G}_{\mathrm{e}}$ and $\stackrel{\leftrightarrow}{G}_{\mathrm{m}}$ as the vacuum propagators of the electric and magnetic fields within the context of a microphysical model, which is an interpretation of the VIE amounting to an extension of Huygens' principle although still an exact solution to Maxwell's equations [21,22]: The particle in this model is envisioned as a collection of differential volume elements within which the internal field is constant. A polarization for each volume element is then defined, which due to the timeharmonic nature of the field will radiate a secondary wave, or wavelet for short. The superposition of all wavelets constitutes the original integral and hence yields the scattered wave at any point in space. Thus, the internal field can be treated as the source of the scattered wave. The advantage of this approach is that effects such as extinction can be understood in terms of the particle properties, size, shape, and refractive index, as they relate to the internal field only. There will be no need to require, for example, that $C^{\text {ext }}$ be considered only in the far-field zone in order to understand its behavior.

The next step is to transform Eq. (13) into an equivalent integral over the particle surface $\mathcal{S}$. This is done using the second vector-dyadic Green's theorem as given in $[23$, p. 300,24 , p. $60-4]$. The result is

$$
\begin{aligned}
& \left.\begin{array}{ll}
\mathbf{E}^{\text {sca }}(\mathbf{r}), & \mathbf{r} \in V^{\text {ext }} \\
-\mathbf{E}^{\text {inc }}(\mathbf{r}), & \mathbf{r} \in V^{\text {int }}
\end{array}\right\}=\oint_{\mathcal{S}}\left\{i \omega \stackrel{\leftrightarrow}{G}_{\mathrm{e}}\left(\mathbf{r}, \mathbf{r}^{\prime}\right)\right. \\
& \times\left[\hat{\mathbf{n}} \times \mathbf{B}^{\text {int }}\left(\mathbf{r}^{\prime}\right)\right]+\stackrel{\leftrightarrow}{G}_{\mathrm{m}}\left(\mathbf{r}, \mathbf{r}^{\prime}\right) \\
& \left.\times\left[\hat{\mathbf{n}} \times \mathbf{E}^{\text {int }}\left(\mathbf{r}^{\prime}\right)\right]\right\} \mathrm{d} S^{\prime} .
\end{aligned}
$$

There is a great deal of meaning contained in Eq. (16) and only a brief discussion of it is given here. When $\mathbf{r}$ is outside of the particle, the $\mathbf{E}^{\text {sca }}$ is given in terms of the tangential component of the electric and magnetic fields on the particle's surface. This formulation for the scattered wave is exact and can be added to the incident wave to yield the total wave, i.e., Eqs. (3) and (4). When $\mathbf{r}$ is inside of the particle however, Eq. (16) produces a wave that exactly cancels the incident wave in $V^{\text {int }}$, see Fig. 5. This is an integral formulation of the EO extinction theorem, see e.g. $[2,24,25]$. The use of the word "extinction" here is customary and refers to the cancellation of the incident wave inside of the particle; EO is not usually associated with $C^{\text {ext }}$, although such association will appear later.

Eq. (16) is valid at any distance from the particle, but Eq. (11) requires the far-field scattering amplitude, which can be obtained from Eq. (16) by taking the $k r \rightarrow \infty$ limit in Green's functions:

$\stackrel{\leftrightarrow}{G}_{\mathrm{e}}\left(\mathbf{r}, \mathbf{r}^{\prime}\right)=\frac{e^{i k r}}{r} \stackrel{\leftrightarrow}{g}_{\mathrm{e}}\left(\hat{\mathbf{r}}, \mathbf{r}^{\prime}\right), \quad k r \rightarrow \infty$

$\stackrel{\leftrightarrow}{G}_{\mathrm{m}}\left(\mathbf{r}, \mathbf{r}^{\prime}\right)=\frac{e^{i k r}}{r} \overleftrightarrow{\mathrm{g}}_{\mathrm{m}}\left(\hat{\mathbf{r}}, \mathbf{r}^{\prime}\right), \quad k r \rightarrow \infty$ 
where

$\overleftrightarrow{g}_{\mathrm{e}}\left(\hat{\mathbf{r}}, \mathbf{r}^{\prime}\right)=\frac{1}{4 \pi}(\stackrel{\leftrightarrow}{I}-\hat{\mathbf{r}} \otimes \hat{\mathbf{r}}) \exp \left(-i k \hat{\mathbf{r}} \cdot \mathbf{r}^{\prime}\right)$

$\overleftrightarrow{g}_{\mathrm{m}}\left(\hat{\mathbf{r}}, \mathbf{r}^{\prime}\right)=\frac{i k}{4 \pi}(\hat{\mathbf{r}} \times \stackrel{\leftrightarrow}{I}) \exp \left(-i k \hat{\mathbf{r}} \cdot \mathbf{r}^{\prime}\right)$

Combining Eqs. (16)-(20), with Eq. (12) in mind, gives

$$
\begin{aligned}
\mathbf{E}_{1}^{\mathrm{sca}}(\hat{\mathbf{r}})= & \oint_{\mathcal{S}}\left\{i \omega \stackrel{\leftrightarrow}{g}_{\mathrm{e}}\left(\hat{\mathbf{r}}, \mathbf{r}^{\prime}\right) \cdot\left[\hat{\mathbf{n}} \times \mathbf{B}^{\mathrm{int}}\left(\mathbf{r}^{\prime}\right)\right]+\stackrel{\leftrightarrow}{g}_{\mathrm{m}}\left(\hat{\mathbf{r}}, \mathbf{r}^{\prime}\right)\right. \\
& \left.\times\left[\hat{\mathbf{n}} \times \mathbf{E}^{\mathrm{int}}\left(\mathbf{r}^{\prime}\right)\right]\right\} \mathrm{d} S^{\prime} .
\end{aligned}
$$

Then, from Eq. (11) the cross section is

$$
\begin{aligned}
C^{\text {ext }}= & \frac{4 \pi}{k\left|\mathbf{E}_{\mathrm{o}}^{\text {inc }}\right|^{2}} \operatorname{Im}\left\{\oint _ { \mathcal { S } } [ \mathbf { E } _ { \mathrm { o } } ^ { \text { inc } } ] ^ { * } \cdot \left[i \omega \stackrel{\leftrightarrow}{g}_{\mathrm{e}}\left(\hat{\mathbf{n}}^{\text {inc }}, \mathbf{r}^{\prime}\right) \cdot\left[\hat{\mathbf{n}} \times \mathbf{B}^{\text {int }}\left(\mathbf{r}^{\prime}\right)\right]\right.\right. \\
& \left.\left.+\stackrel{\leftrightarrow}{g}_{\mathrm{m}}\left(\hat{\mathbf{n}}^{\text {inc }}, \mathbf{r}^{\prime}\right) \cdot\left[\hat{\mathbf{n}} \times \mathbf{E}^{\text {int }}\left(\mathbf{r}^{\prime}\right)\right]\right] \mathrm{d} S^{\prime}\right\} .
\end{aligned}
$$

Although this expression is obtained using the far-field approximation, the use of the optical theorem makes Eq. (22) exact, see [15]. Therefore, the conclusions predicated upon Eq. (22) in the following, and the close relatives Eqs. (23) and (27), are not restricted to the farfield zone.

\subsection{The partial surface}

Eq. (22) can show how different regions of the particle surface contribute to $C^{\text {ext }}$. To do this, the integral is evaluated only over the portion of $\mathcal{S}$ extending from $\theta=\pi$ to $\theta_{s}$, where $\theta$ is the polar angle. This partial surface, denoted $\partial \mathcal{S}$, is shown in Fig. 2 where the particle is divided into its geometrically illuminated and shaded sides, $\mathcal{S}^{\text {ill }}$ and $\mathcal{S}^{\text {sha }}$, respectively. Evaluating Eq. (22) over $\partial \mathcal{S}$ and using Eq. (9), a partial extinction efficiency factor is defined as

$$
\begin{aligned}
\partial Q^{\text {ext }}\left(\theta_{s}\right)= & \frac{1}{C^{\text {geo }}} \frac{4 \pi}{k\left|\mathbf{E}_{\mathrm{o}}^{\text {inc }}\right|^{2}} \operatorname{Im}\left\{\int _ { \partial \mathcal { S } } [ \mathbf { E } _ { \mathrm { o } } ^ { \text { inc } } ] ^ { * } \cdot \left[i \omega \stackrel{\leftrightarrow}{\mathrm{g}}_{\mathrm{e}}\left(\hat{\mathbf{n}}^{\mathrm{inc}}, \mathbf{r}^{\prime}\right) \cdot\left[\hat{\mathbf{n}} \times \mathbf{B}^{\mathrm{int}}\left(\mathbf{r}^{\prime}\right)\right]\right.\right. \\
& \left.\left.+\stackrel{\leftrightarrow}{\mathrm{g}}_{\mathrm{m}}\left(\hat{\mathbf{n}}^{\mathrm{inc}}, \mathbf{r}^{\prime}\right) \cdot\left[\hat{\mathbf{n}} \times \mathbf{E}^{\mathrm{int}}\left(\mathbf{r}^{\prime}\right)\right]\right] \mathrm{d} S^{\prime}\right\} .
\end{aligned}
$$

When $\theta_{s}=\pi$, there is no integration surface and Eq. (23) is zero, whereas when $\theta_{s}=0$, the partial surface becomes the complete particle surface $\partial \mathcal{S}=\mathcal{S}$ and Eq. (23) yields

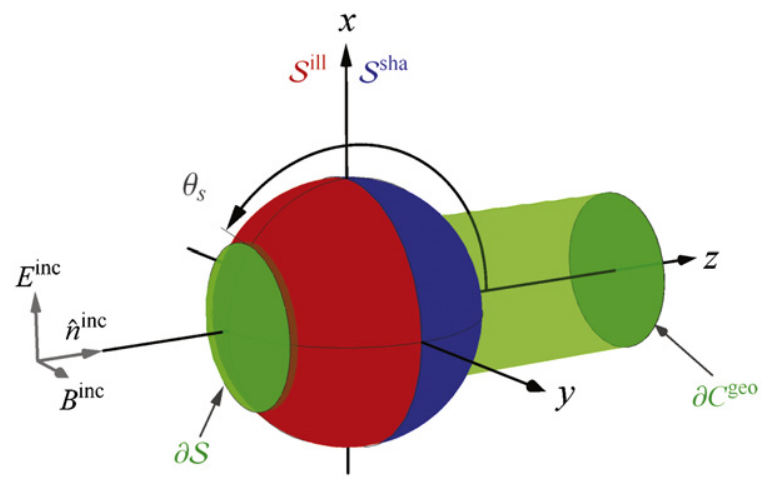

Fig. 2. Partial surface $\partial \mathcal{S}$, which is used to calculate $\partial Q^{\text {ext }}$ of Eq. (23) and $\partial C^{\text {geo }}$ of Eq. (24). The geometrically illuminated and shaded sides of the particle, $\mathcal{S}^{\text {ill }}$ and $\mathcal{S}^{\text {sha }}$, are shown in red and blue.
$Q^{\text {ext }}$. Thus, $\theta_{s}$ closes the surface as it decreases from $\pi$ to zero. Also needed in the following is the projection of $\partial \mathcal{S}$ into the forward direction,

$\partial C^{\text {geo }}\left(\theta_{S}\right)=-\int_{\partial \mathcal{S}} \hat{\mathbf{n}} \cdot \hat{\mathbf{n}}^{\text {inc }} \mathrm{d} S$,

which is shown in Fig. 2. The negative sign in Eq. (24) is included so that $\partial C^{\text {geo }}$ is a positive quantity. Note that $\partial Q^{\text {ext }}$ is not intended to represent a physical quantity until $\theta_{s}=0$; the point here is to illustrate how different regions of the particle contribute to the eventual value of $C^{\text {ext }}$.

Now consider Fig. 3, which shows $\partial Q^{\text {ext }}$ as a function of decreasing $\theta_{s}$, hence increasing $\partial \mathcal{S}$, for particles with various $k R$ and $m$. These values fall into three categories quantified by the phase shift parameter,

$\rho=2 k R \operatorname{Re}\{m-1\}$.

One can think of $\rho$ as a measure of the degree of refraction occurring throughout the particle [3, p. 132]. The three categories $\rho \ll 1,1 \lesssim \rho \lesssim 10$, and $\rho \gg 1$, represent the weak, intermediate, and strong refraction regimes, respectively. A particle can be strongly refractive in this sense even if its refractive index is close to one (e.g., $m=1.05+0 i$ ) provided that its size parameter is sufficiently large.

Each curve in Fig. 3 rises from zero at $\theta_{s}=\pi$ to near two when $\theta_{s}=\pi / 2$. However, as $\theta_{s}$ decreases past $\pi / 2$ on its way to zero, the curves separate into three groups coincident with the three categories of $\rho$. For $\rho \ll 1$, the curves decrease from two back to a small value for $Q^{\text {ext }}$ as $\theta_{s} \rightarrow 0$. If $1 \lesssim \rho \lesssim 10$, the curves spread. Lastly, if $\rho \gg 1$, the curves bundle together and roughly hold a constant value of $\partial Q^{\text {ext }} \sim 2$ as $\theta_{s} \rightarrow 0$. For comparison, the values of $Q^{\text {ext }}$ for each particle as calculated directly from the coefficients of the Mie series are shown in the legend. These values are consistent with the curves' $\theta_{s}$ intercepts with the vertical axis.

An important conclusion from Fig. 3 is that when $\rho \gg 1$, the geometrically illuminated portion of the particle appears to be solely responsible for the value of the cross section. The shaded portion appears not to contribute significantly. Moreover, this behavior is largely insensitive to $k R$ and $m$ independently; it depends on them only through their combination in $\rho$. An especially striking example is to compare the $k R=1000, m=1.05+0 i$ curve to the $k R=125.0$, $m=1.40+0.1 i$ curve. This first particle is very large and highly transparent while the second is smaller, very absorbent, and thus opaque. Yet $Q^{\text {ext }} \simeq 2$ for both particles, and moreover, their curves reveal that the illuminated surface appears solely responsible for this value.

\subsection{Further insight from a surface map}

A formulation similar to Eq. (23) can reveal how the $\rho$-evolution of the internal field is correlated with the cross section. Begin by dividing the particle surface into differential elements $\Delta S_{i}$ in Eq. (21). This gives

$$
\begin{aligned}
\mathbf{E}_{1}^{\mathrm{sca}}(\hat{\mathbf{r}})= & \sum_{i}\left\{i \omega \stackrel{\leftrightarrow}{g}_{\mathrm{e}}\left(\hat{\mathbf{r}}, \mathbf{r}_{i}\right) \cdot\left[\hat{\mathbf{n}} \times \mathbf{B}^{\mathrm{int}}\left(\mathbf{r}_{i}\right)\right]+\stackrel{\leftrightarrow}{\mathrm{g}}_{\mathrm{m}}\left(\hat{\mathbf{r}}, \mathbf{r}_{i}\right)\right. \\
& \left.\times\left[\hat{\mathbf{n}} \times \mathbf{E}^{\mathrm{int}}\left(\mathbf{r}_{i}\right)\right]\right\} \Delta S_{i}, \quad \Delta S_{i} \rightarrow 0 .
\end{aligned}
$$




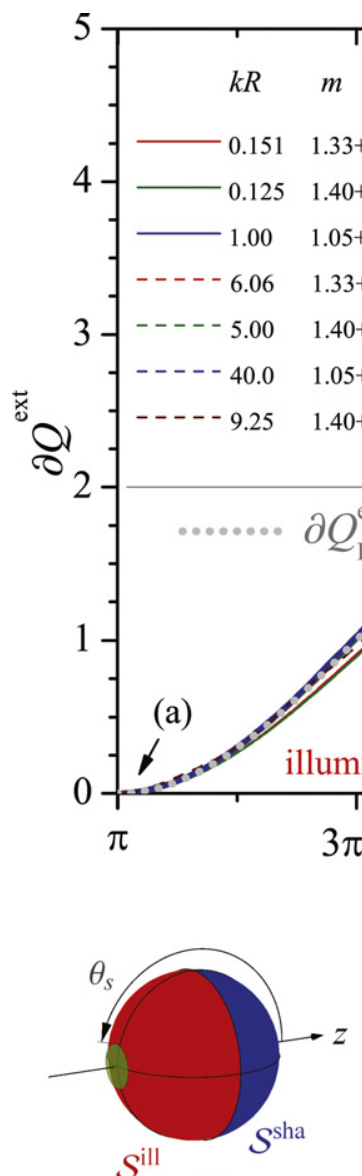

(a)

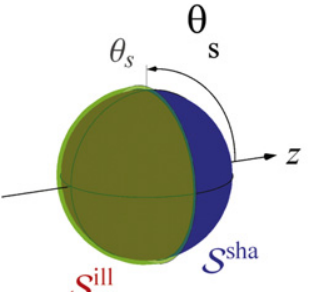

(b)

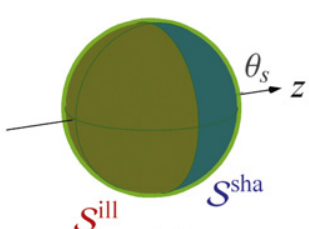

(c)

Fig. 3. Plot of $\partial Q^{\text {ext }}$ for various spherical particles as a function of $\theta_{s}$, recall Fig. 2. The values of $k R$, $m, \rho$, and $Q^{\text {ext }}$ as calculated from the Mie series coefficients, are shown for each particle in the legend. Also shown is $\partial Q_{\mathrm{PC}}^{\mathrm{ext}}$ corresponding to the perfectly conducting particle discussed in Section 4.5 . To aid interpretation, diagrams of the integration surface $\partial \mathcal{S}$ are shown, labeled (a)-(c), corresponding to the similarly labeled regions in the plot.

Then $Q^{\text {ext }}$ can be expressed in terms of this scattering amplitude as the product $\left[\mathbf{E}_{\mathrm{o}}^{\mathrm{inc}}\right]^{*} \cdot \mathbf{E}_{1}^{\text {sca }}$ evaluated in the forward direction via Eq. (11). Phase shifts, $\Delta z_{i}$, are then defined by this product as

$$
\begin{aligned}
\Delta z_{i}(\hat{\mathbf{r}})= & {\left[\mathbf{E}_{\mathrm{o}}^{\mathrm{inc}}\left(\mathbf{r}_{i}\right)\right]^{*} \cdot\left\{i \omega \stackrel{\leftrightarrow}{g}_{\mathrm{e}}\left(\hat{\mathbf{r}}, \mathbf{r}_{i}\right) \cdot\left[\hat{\mathbf{n}} \times \mathbf{B}^{\mathrm{int}}\left(\mathbf{r}_{i}\right)\right]\right.} \\
& \left.+\stackrel{\leftrightarrow}{g}_{\mathrm{m}}\left(\hat{\mathbf{r}}, \mathbf{r}_{i}\right) \cdot\left[\hat{\mathbf{n}} \times \mathbf{E}^{\mathrm{int}}\left(\mathbf{r}_{i}\right)\right]\right\} \Delta S_{i},
\end{aligned}
$$

which through Eq. (11) gives the efficiency,

$Q^{\text {ext }}=\frac{1}{C^{\text {geo }}} \frac{4 \pi}{k\left|\mathbf{E}_{\mathrm{o}}^{\text {inc }}\right|^{2}} \operatorname{Im}\left\{\sum_{i} \Delta z_{i}\left(\hat{\mathbf{n}}^{\text {inc }}\right)\right\}$.

Eq. (28) has a simple meaning using a particle-surface analog of the microphysical model mentioned in Section 4. Each surface element in Eq. (26) can be associated with a surface source that radiates a wavelet from its location $\mathbf{r}_{i}$ to $\mathbf{r}$. The quantities $\Delta z_{i}$ then represent the phase shift between a wavelet's surface source and the incident wave at that source's location on the particle.

Fig. 4 is a color-coded particle-surface map showing how these incident-field-relative wavelet phase-shifts $\Delta z_{i}$ evolve as a function of $\rho$. In (a) and (b), the geometrically illuminated and shaded sides display opposing uniform phase shifts of $\pi / 2$ and $-\pi / 2$, respectively. In the sum in Eq. (28), this means that the contribution to $Q^{\text {ext }}$ made by the illuminated side is mostly canceled by that of the shaded side, which is consistent with the behavior seen for the $\rho \ll 1$ curves in Fig. 3. In (c) and (d), the illuminated side also shows a phase shift of roughly $\pi / 2$ whereas the shaded side is more varied. This variation prevents that side from as effectively canceling the contribution made by the illuminated side. Thus, a larger value for $Q^{\text {ext }}$ results, again consistent with Fig. 3. Now consider (e) and (f), which show the $\rho \gg 1$ particle. Here again the illuminated side's phase shift is primarily a constant $\pi / 2$, whereas the shaded side now displays rapid variations covering the entire range from $-\pi$ to $\pi$. This rapid variation washes-out the shaded side's contribution to $Q^{\text {ext }}$ in Eq. (28).

\subsection{Illuminated and shaded hemispheres}

Eq. (16) is the EO extinction theorem. The same integral, discretized, appears in its far-field form in 
a

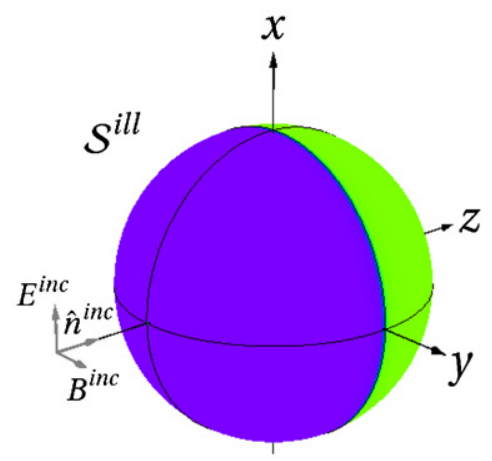

C

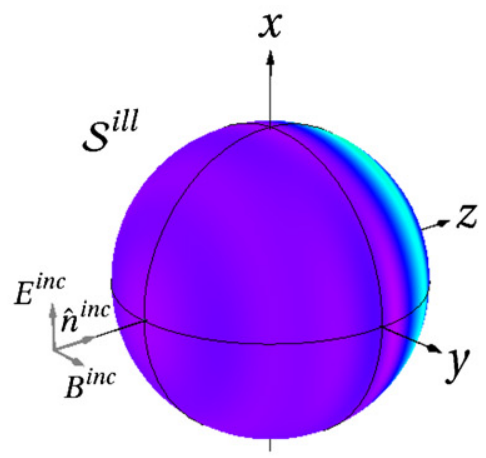

e

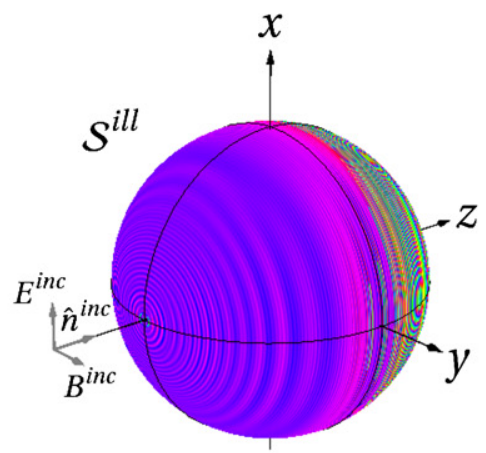

b

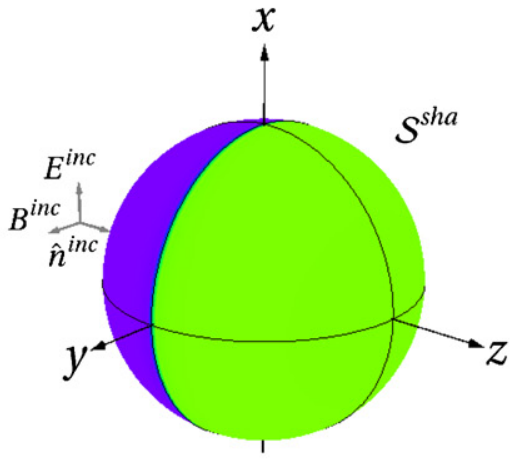

d

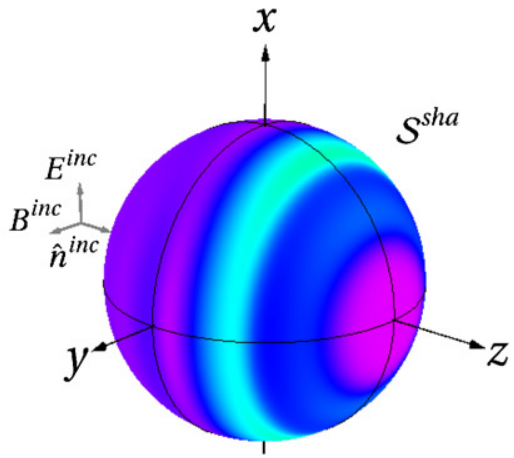

$f$

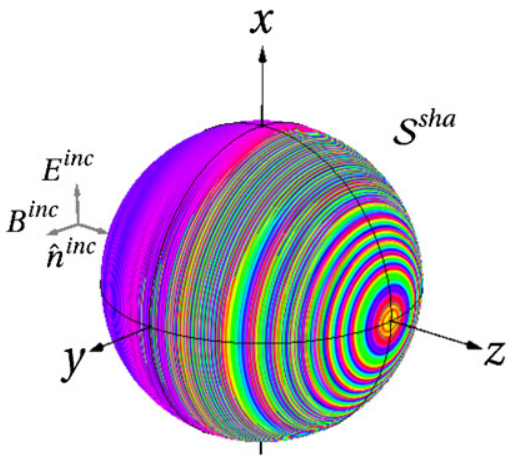

phase angle

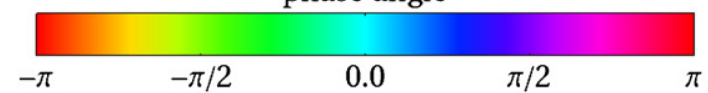

Fig. 4. Evolution of the incident-field-relative wavelet phase shifts $\Delta z_{i}$, Eq. (27), as a function of $\rho$. Each pair shows views of the $\mathcal{S}^{\text {ill }}$ and $\mathcal{S}^{\text {sha }}$ sides of the particle. The surface is color coded to display these shifts. The refractive index is $m=1.33+0 i$. Pair (a) and (b) show a $\rho=0.1$ particle where $k R=0.151$. Pairs (c)-(d) and (e)-(f) show particles with $\rho=4.0$ and $\rho=100$ where $k R=6.06$ and $k R=151$, respectively. Each of these particles is included in Fig. 3 .

Eq. (27) to give $Q^{\text {ext }}$ through Eq. (28), which suggests a connection between EO and $Q^{\text {ext }}$. Figs. 5 and 6 investigate this connection.

Each plot in Fig. 5 shows the $x-z$ plane passing through the same $\rho=4$ particle in Fig. 4. The purpose of this figure is to demonstrate the dual role of the surface integral in Eq. (16). To do this, the magnitude of the superposition of two fields is shown. One of the fields is the incident, $\mathbf{E}^{\mathrm{inc}}$. The other is either $\mathbf{E}^{\text {ill }}$ or $\mathbf{E}^{\text {sha }}$, which respectively, are the fields produced by Eq. (16) when the surface integral is evaluated over $\mathcal{S}^{\text {ill }}$ or $\mathcal{S}^{\text {sha }}$ only, i.e.,

$$
\mathbf{E}^{\mathrm{ill}}(\mathbf{r})=\int_{\mathcal{S}^{\mathrm{ill}}}\left\{i \omega \stackrel{\leftrightarrow}{G}_{\mathrm{e}}\left(\mathbf{r}, \mathbf{r}^{\prime}\right) \cdot\left[\hat{\mathbf{n}} \times \mathbf{B}^{\text {int }}\left(\mathbf{r}^{\prime}\right)\right]+\stackrel{\leftrightarrow}{G}_{\mathrm{m}}\left(\mathbf{r}, \mathbf{r}^{\prime}\right) \cdot\left[\hat{\mathbf{n}} \times \mathbf{E}^{\text {int }}\left(\mathbf{r}^{\prime}\right)\right]\right\} \mathrm{d} S^{\prime}
$$

and

$$
\begin{aligned}
\mathbf{E}^{\text {sha }}(\mathbf{r})= & \int_{\mathcal{S}^{\text {sha }}}\left\{i \omega \stackrel{\leftrightarrow}{G}_{\mathrm{e}}\left(\mathbf{r}, \mathbf{r}^{\prime}\right) \cdot\left[\hat{\mathbf{n}} \times \mathbf{B}^{\mathrm{int}}\left(\mathbf{r}^{\prime}\right)\right]\right. \\
& \left.+\overleftrightarrow{G}_{\mathrm{m}}\left(\mathbf{r}, \mathbf{r}^{\prime}\right) \cdot\left[\hat{\mathbf{n}} \times \mathbf{E}^{\mathrm{int}}\left(\mathbf{r}^{\prime}\right)\right]\right\} \mathrm{d} S^{\prime}
\end{aligned}
$$


a

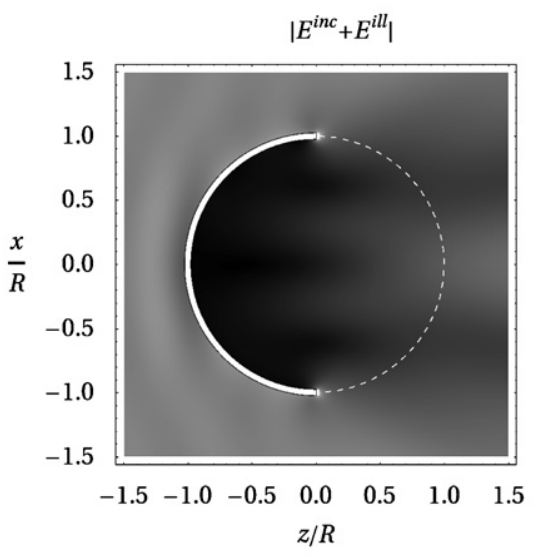

b

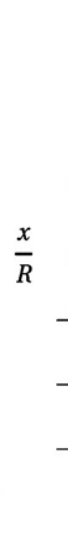

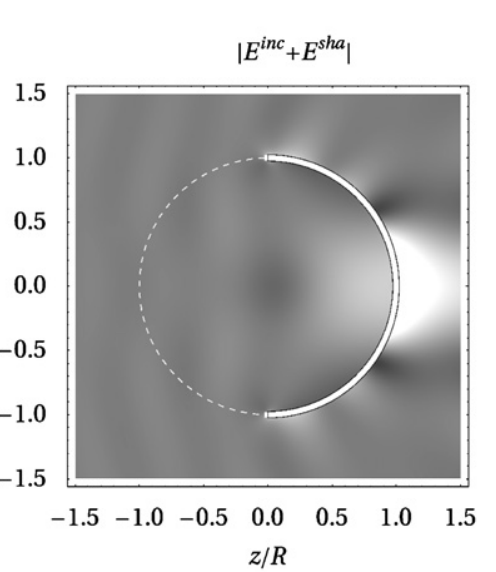

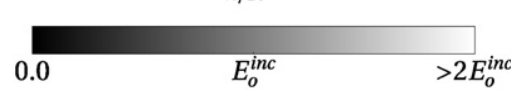

C

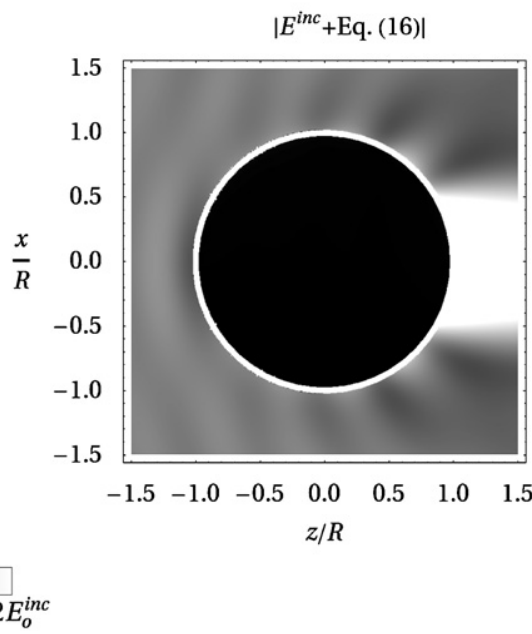

Fig. 5. Ewald-Oseen extinction theorem in action. Plots (a) and (b) show the magnitude of the superposition of the incident field $\mathbf{E}^{\text {inc }}$ and $\mathbf{E}^{\text {ill }}$ or $\mathbf{E}^{\text {sha }}$ given by Eqs. (29) or (30). Together, Eqs. (29) and (30) are equivalent to the EO theorem, Eq. (16). In (a), $\mathbf{E}^{\text {ill }}$ is produced by the particle's illuminated side $\mathcal{S}^{\text {ill }}$, denoted by the white arc. In (b), $\mathbf{E}^{\text {sha }}$ is produced by the shaded side $\mathcal{S}^{\text {sha }}$. Together, plots (a) and (b) yield (c). The particle is the same $\rho=4.0$ particle in Figs. 4 and 6.

Together, Eqs. (29) and (30) are equivalent to Eq. (16). Plot (a) in Fig. 5 shows the magnitude of the field superposition corresponding to the illuminated side, i.e., $\left|\mathbf{E}^{\text {inc }}+\mathbf{E}^{\text {ill }}\right|$. The thick white arc indicates the integration surface $\mathcal{S}^{\text {ill }}$ and is an excluded region due to the singularity occurring in the integral when $\mathbf{r}$ approaches $\mathcal{S}^{\text {ill }}$ in Eq. (29). In other words, this arc denotes the surface source on $\mathcal{S}^{\text {ill }}$. The dashed white arc denotes the rest of the particle surface, which is not evaluated in Eq. (29). Plot (b) shows the analogous superposition for the shaded side, i.e., $\left|\mathbf{E}^{\text {inc }}+\mathbf{E}^{\text {sha }}\right|$. Together, plots (a) and (b) yield (c), where the superposition corresponding to the full surface integral is shown, i.e., $\mid \mathbf{E}^{\text {inc }}+$ Eq. (16)|. The interior of the particle is black in plot (c), which since the incident field is present everywhere indicates that the field produced by the surface integral in Eq. (16) cancels the incident field. Outside the particle, this plot shows the superposition of the incident and scattered fields, i.e., the total field. In general, both surfaces of the particle are required to fully cancel the incident field inside.

Fig. 6 shows the same field superposition as in (a) and (b) of Fig. 5, except here all three $\rho$ values of Fig. 4 are considered. Plots (a) and (b), which show the $\rho=0.1$ particle, are very similar demonstrating that the sources on both sides of the particle contribute significantly to the surface integral of Eq. (16). Next consider (c) and (d) in Fig. 6 , which show the $\rho=4.0$ particle. In (c), the particle's interior region cupped by $\mathcal{S}^{\text {ill }}$ is mostly black, indicating that the field of Eq. (29) mostly cancels the incident field there. Quite the opposite behavior is seen in (d). Lastly, consider (e) and (f) in Fig. 6, which shows the $\rho=100$ particle. Plot (e) reveals that the illuminated-hemisphere's source nearly cancels the incident field throughout the entire particle. This is seen by the mostly black appearance of the interior. The shaded-hemisphere source however, leaves the interior essentially unaffected with only the incident field seen there.

The evolution with $\rho$ displayed in Fig. 6 reveals that the source on $\mathcal{S}^{\text {ill }}$ would exactly cancel the incident field throughout the entire particle in the $\rho \rightarrow \infty$ limit. In this same limit, the shaded side would make no contribution to the particle's interior but would contribute strongly outside. Meanwhile, Figs. 3 and 4 demonstrate that the illuminated side determines the value of $Q^{\text {ext }}$ while the shaded side contributes nothing in this same limit. The next section will consider the special case of a perfectly conducting particle where, when combined with these results above, it will finally become clear how EO cancellation is responsible for the paradox.

\subsection{Perfectly conducting particle: a revealing special case}

For a very large perfectly conducting (PC) particle, the mathematics of the above become simplified, while at the same time the $\rho \gg 1$ regime is obtained. A surface current $\mathbf{K}$ resides on such a particle given by the tangential component of the magnetic field [26,27],

$\hat{\mathbf{n}} \times \mathbf{B}^{\text {inc }}(\mathbf{r})+\hat{\mathbf{n}} \times \mathbf{B}^{\text {sca }}(\mathbf{r})=\mu_{\mathrm{o}} \mathbf{K}(\mathbf{r}), \quad \mathbf{r} \in \mathcal{S}$.

Along with the recognition that the tangential electric field is zero on $\mathcal{S}$, Eq. (16) can be used to find the field radiated by this surface current as

$$
\left.\begin{array}{ll}
\mathbf{E}^{\text {sca }}(\mathbf{r}), & \mathbf{r} \in V^{\text {ext }} \\
-\mathbf{E}^{\text {inc }}(\mathbf{r}), & \mathbf{r} \in V^{\text {int }}
\end{array}\right\}=i \omega \mu_{\mathrm{o}} \oint_{\mathcal{S}} \overleftrightarrow{G}_{\mathrm{e}}\left(\mathbf{r}, \mathbf{r}^{\prime}\right) \cdot \mathbf{K}\left(\mathbf{r}^{\prime}\right) \mathrm{d} S^{\prime} \quad(\mathrm{PC})
$$

see [23, p. 64]. In general, current is induced over the entire surface. However, if the particle is very large the current over $\mathcal{S}^{\text {sha }}$ becomes negligible compared to that on 
a

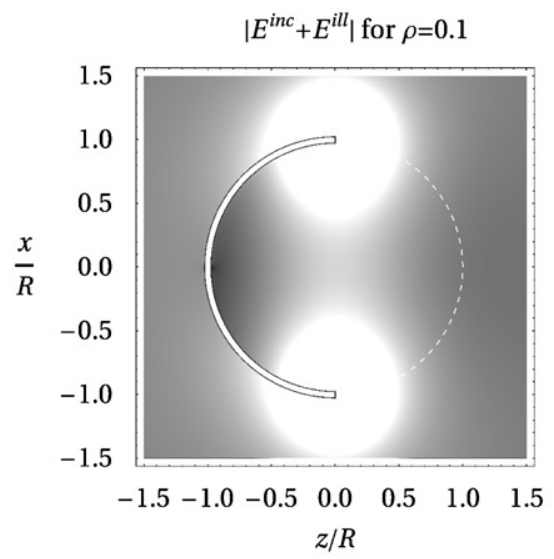

C

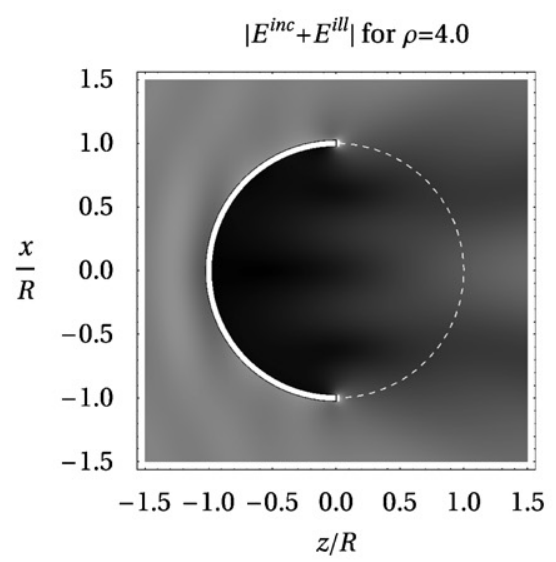

e

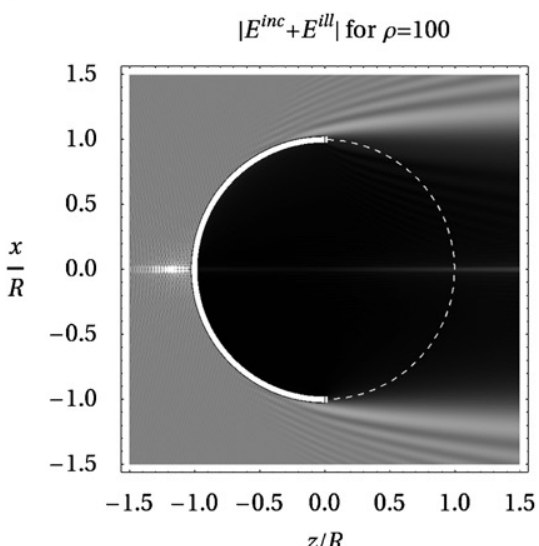

b

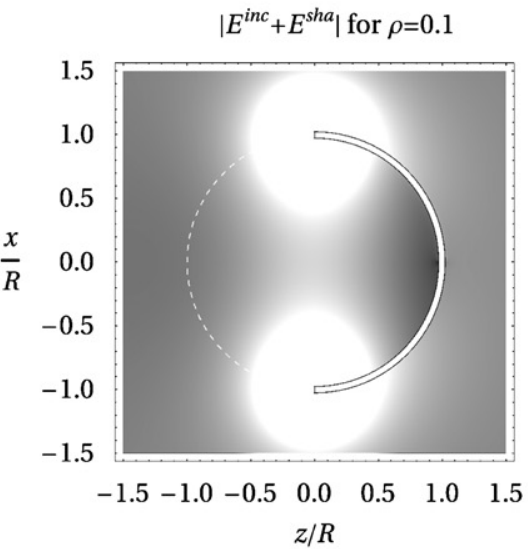

d

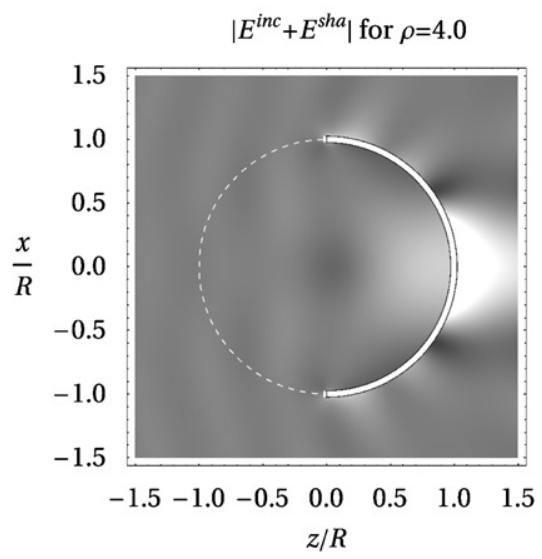

f

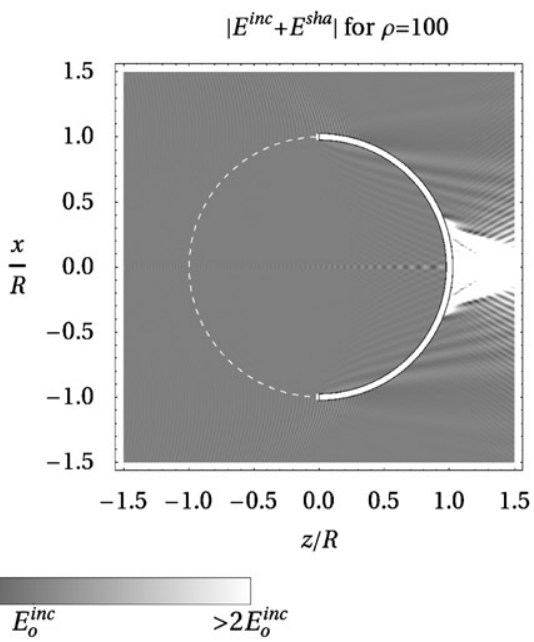

Fig. 6. Magnitude of the superpositions of $\mathbf{E}^{\text {inc }}$ and Eqs. (29) or (30) described in Fig. 5. The particles are the same dielectric particles in Figs. 4 and 5.

$\mathcal{S}^{\text {ill }}$ [30]. The physical optics approximation can then be introduced, which treats each differential surface element as an infinite plane, such that $[28,29]$

$\hat{\mathbf{n}} \times \mathbf{B}^{\mathrm{sca}}(\mathbf{r})=\hat{\mathbf{n}} \times \mathbf{B}^{\mathrm{inc}}(\mathbf{r}), \quad \mathbf{r} \in \mathcal{S}^{\mathrm{ill}}, k R \rightarrow \infty$.
Using Eqs. (1), (2), and (33) in combination with Eq. (31), gives

$$
\mathbf{K}(\mathbf{r})= \begin{cases}2 k\left(\omega \mu_{\mathrm{o}}\right)^{-1} \hat{\mathbf{n}} \times\left[\hat{\mathbf{n}}^{\mathrm{inc}} \times \mathbf{E}^{\mathrm{inc}}(\mathbf{r})\right], & \mathbf{r} \in \mathcal{S}^{\text {ill }}, \\ 0, & \mathbf{r} \in \mathcal{S}^{\text {sha }} .\end{cases}
$$


Combining Eqs. (32) and (34), using the far-field form of Green's function in Eqs. (19) and (11), one finds

$C^{\text {ext }}=2 \operatorname{Im}\left\{-i \int_{\mathcal{S}^{\text {ill }}} \hat{\mathbf{n}} \cdot \hat{\mathbf{n}}^{\text {inc }} \mathrm{d} S\right\}=2 C^{\text {geo }}$

as expected, where Eq. (24) has been used with $\theta_{s}=\pi / 2$, i.e., $\partial C^{\text {geo }}(\pi / 2)=C^{\text {geo }}$.

The calculation leading to Eq. (35) is simple enough to see the significance of the factor of two in the paradox. The first appearance of this factor is in the surface current Eq. (34). This form for the current is a consequence of the boundary conditions and the requirement that the total wave inside the particle is zero. Consideration of Eqs. (31), (33), and (34) show that the tangential component of the total magnetic field on $\mathcal{S}^{\text {ill }}$ is twice that of the same field component that would exist at those points in space in the particle's absence, i.e., twice that of the incident magnetic field. This factor of two is required so that the current can radiate a wave into the particle that cancels the incident wave there, and simultaneously radiate a scattered wave of similar magnitude outside to satisfy energy conservation.

\subsection{Revelation of the Ewald-Oseen origin of the paradox}

To investigate the similarities between the perfectly conducting (PC) particle and the dielectric ones earlier, consider the PC analog to Eq. (23), which will be denoted $Q_{P C}^{\text {ext }}$. This PC particle partial-efficiency-factor is given by Eqs. (9) and (35), with the latter being evaluated over $\partial \mathcal{S}$. Fig. 3 shows $\partial Q_{\mathrm{PC}}^{\mathrm{ext}}$ plotted along with the dielectric particles. The behavior of $\partial Q_{\mathrm{PC}}^{\mathrm{ext}}$ in relation to the others for $\rho \gg 1$ shows a strong functional similarity, implying the equivalence of the following equations from which these curves are derived:

$$
\begin{aligned}
C^{\text {ext }}= & \frac{4 \pi}{k\left|\mathbf{E}_{o}^{\text {inc }}\right|^{2}} \operatorname{Im}\left\{\oint _ { \mathcal { S } } [ \mathbf { E } _ { \mathrm { o } } ^ { \text { inc } } ] ^ { * } \cdot \left[i \omega \stackrel{\leftrightarrow}{g}_{\mathrm{e}}\left(\hat{\mathbf{n}}^{\text {inc }}, \mathbf{r}^{\prime}\right) \cdot\left[\hat{\mathbf{n}} \times \mathbf{B}^{\text {int }}\left(\mathbf{r}^{\prime}\right)\right]\right.\right. \\
& \left.\left.+\stackrel{\leftrightarrow}{g}_{\mathrm{m}}\left(\hat{\mathbf{n}}^{\text {inc }}, \mathbf{r}^{\prime}\right) \cdot\left[\hat{\mathbf{n}} \times \mathbf{E}^{\text {int }}\left(\mathbf{r}^{\prime}\right)\right]\right] \mathrm{d} S^{\prime}\right\}=2 C^{\text {geo }}, \quad \rho \rightarrow \infty
\end{aligned}
$$

$C^{\text {ext }}=2 \operatorname{Im}\left\{-i \int_{\mathcal{S}^{\text {ill }}} \hat{\mathbf{n}} \cdot \hat{\mathbf{n}}^{\text {inc }} \mathrm{d} S\right\}=2 C^{\text {geo }}, \quad(\mathrm{PC}), \quad k R \rightarrow \infty$.

Eq. (36) gives $C^{\text {ext }}$ for a dielectric particle as a complicated integral of the tangential surface-field components. Eq. (37) is much simpler, giving $C^{\text {ext }}$ for a large PC particle in terms of its projected area $C^{\text {geo }}$. The equivalence of these formulations, as suggested by the coincidence of the $\partial Q^{\text {ext }}$ and $\partial Q_{\mathrm{PC}}^{\text {ext }}$ curves in Fig. 3, is remarkable because it implies that $C^{\text {ext }} \rightarrow 2 C^{\text {geo }}$ for dielectric particles for the same reasons as it does for large PC particles. As further evidence for this similarity, Fig. 6 shows that the portion of the surface source responsible for EO cancellation resides only on $\mathcal{S}^{\text {ill }}$ if $\rho \gg 1$, which is also the behavior seen for the PC particle. Fig. 4 shows that the reason the shaded-side source does not contribute to this cancellation is because its phase varies rapidly across $\mathcal{S}^{\text {sha }}$ due to the high degree of refraction. Again, this is consistent with the PC particle, where the surface source is simply zero across $\mathcal{S}^{\text {sha }}$.

It is now finally possible to explain the extinction behavior associated with the paradox as being a consequence of the following conditions:

1. The EO theorem requires that the incident wave must be canceled everywhere inside a particle.

2. This cancellation is achieved by secondary radiation from a surface source derived from the particle's own internal field. This same source also accounts for the external scattered wave.

3. If $\rho \gg 1$, the degree of refraction in the particle is large and the portion of the source responsible for EO cancellation resides on the particle's geometrically illuminated surface.

Condition 1 explains why the paradox is observed for any particle shape, not just the spheres considered here. Conditions 2 and 3 explain why there is a factor of two in the paradox: Since the EO cancellation wave and the external scattered wave are produced by the same source in the particle, they are fundamentally connected. The cancellation wave must be associated with a net energy flow of $I^{\text {inc }} C$ geo through $\mathcal{S}$ en otherwise it could not cancel the incident wave. At the same time, the energy missing as a consequence of this cancellation must appear elsewhere, which is achieved outside of the particle by the scattered wave, thus supplying another factor of $I^{\text {inc }} C^{\text {geo }}$. Condition 3 shows why only the geometrically illuminated side of a particle is involved. After all, EO cancellation must begin across this surface and extends throughout the particle along the propagation direction of the incident wave. If $\rho$ is not large, then the surface source across the whole particle is needed to achieve EO cancellation. This fact is seen in Fig. 5. Fig. 6 shows that when $\rho$ is large, the shaded side does not participate in this cancellation. However, this is not because the surface source is zero in that region. Rather, it is because strong refraction occurring throughout the particle varies the phase of this source relative to the incident wave so wildly that its contribution to $C^{\text {ext }}$ is washed out.

\section{Revisiting the canonical explanations of the paradox}

One typically encounters variations of two explanations for the paradox; the diffraction explanation of Section 2, and an alternative based on near-field shadow formation, see $[3,4]$, respectively. Using either one is able to predict $C^{\text {ext }}$ for large particles of any shape under plane wave illumination. In this regard these explanations are useful. In the following, both are briefly examined where subtle problems with them are revealed. Collectively, these problems are powerful evidence that neither explanation can correctly explain why $C^{\text {ext }}$ attains its asymptotic value of $2 C^{\text {geo }}$. Moreover, one will see that the EO explanation does not suffer from these problems and thus constitutes a more general explanation for the paradox. 


\subsection{Diffraction explanation}

This explanation argues that diffraction from a large particle's shadow boundary explains the paradox. A critical assumption here is that the incident wave can be meaningfully separated into portions that are intercepted by the particle and those not. This separation is reasonable if the particle's transverse dimensions are much greater than $\lambda$. However, consider Fig. 7 where $Q^{\text {ext }}$ is shown as a function of $\rho$ when both the particle size and refractive index are varied, but the wavenumber $k$ is fixed. Here each curve tends to two, but notice that the top two curves, where $k R=10$, approaches $Q^{\mathrm{ext}}=2$ as $\operatorname{Re}\{m\}$ is increased from 1.0 to 4.0 . The only difference between these curves is that the top one has absorption $\operatorname{Im}\{m\}=0.03$ while the middle does not. This important figure demonstrates that the paradox occurs in the $\rho \gg 1$ limit; it is not just a large size parameter effect. In other words, $C^{\text {ext }} \rightarrow 2 C^{\text {geo }}$ for a small particle provided that $m$ is large enough that $\rho \gg 1$.

Fig. 7 calls into question the validity of van de Hulst's ray-based wavefront separation. Of course, it is always possible to identify the portion of wavefront geometrically intercepted by a particle regardless of $k R$ or $m$. But, it is only in the large size regime that the energy flow contained in these respective portions of the wavefront can be thought of as pursuing separate paths, distinct from each other, and thus making distinctly separate contributions to $C^{\text {ext }}$. If the particle is small, e.g., $k R \lesssim 10$, then it is not meaningful to identify a bundle of rays that intercept the particle. The reason is the energy flow in a portion of the wavefront with transverse area $C^{\text {geo }}$ undergoes ray-like propagation only if the dimensions of that area are much greater than $\lambda[3, p$. 21]. Consequently, van de Hulst adds that “...the [wavefront] separation described in this section is strictly only possible if the particle is very large..." [3, p. 104]. Hence even van de Hulst would agree that the diffraction explanation does not predict $C^{\text {ext }} \rightarrow 2 C^{\text {geo }}$ for the small $k R=10$ highly refractive particles in Fig. 7 .

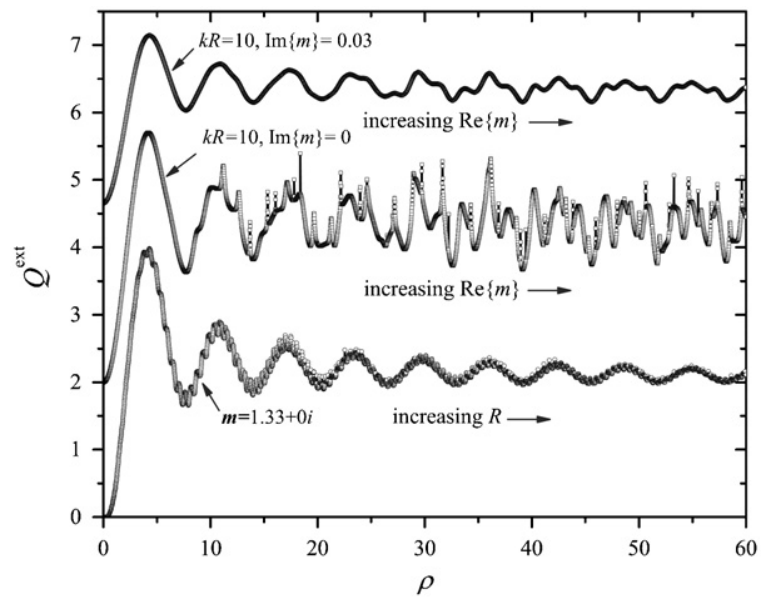

Fig. 7. Efficiency factor $Q^{\text {ext }}$ for particles as a function of $\rho$. The wavenumber is constant for all curves. The vertical scale corresponds to the bottom curve and the others are shifted up by additive factors of two for clarity.
Nevertheless, suppose one insists that the diffraction explanation need not require the concept of intercepted rays, just that a portion $C^{\text {geo }}$ of the incident wave front is subtended by the particle, and thus, its energy flow is somehow removed from the beam. Then, when this is added to the contribution from diffraction, there would be a total of $C^{\text {ext }}=2 C^{\text {geo }}$, as wanted. Unfortunately, this argument fails since one would find that $C^{\text {ext }}=2 C^{\text {geo }}$ independent of particle size or refractive index. In other words, the argument yields $C^{\text {ext }}=2 C^{\text {geo }}$ even for $\rho \ll 1$; a behavior that is not seen in the exact Mie calculations of Fig. 7.

As mentioned in Section 2, the diffraction explanation assumes that $C^{\text {ext }}$ is calculated, or measured, in the farfield zone. The reason is that only in the far-field can diffraction divert enough energy flow from the forward direction to account for a total contribution of $C^{\text {geo }}$ to $C^{\text {ext }}$. For example, the classic text by Born and Wolf says [2]; "In addition to the light intercepted by the obstacle (lost by reflection and absorption), with cross section $C^{\text {geo }}$, there is an additional contribution to the extinction, arising from the neighborhood of the edge of the shadow, and its contribution is evidently [italics added] also equal to $C^{\text {geo }}$. In order to verify [this] relation by experiment one must collect the light over a sufficiently wide area and far enough away from the obstacle." However, in disagreement with Born and Wolf, a complete explanation must be able to apply in both the near and far-field zones. This is because the value of $C^{\text {ext }}$ must be independent of the distance from the particle at which it is calculated or (properly) measured. In short, the reason for this is because $C^{\text {sca }}, C^{\text {abs }}, C^{\text {ext }}$ are collectively a statement of energy conservation, and as such, cannot depend on an assumed (far-field) distance from the particle, e.g. see [17, Section 2.8]. This fact is derived analytically for any particle in [15]. The diffraction explanation is able to quantitatively find that $C^{\text {ext }} \rightarrow 2 C^{\text {geo }}$ by integrating the Fraunhofer diffraction pattern [1,3]. But, Fraunhofer diffraction is by definition a far-field concept. As one moves infinitesimally close to the particle's surface, the diversion of energy flow from the forward direction due to this diffraction vanishes.

\subsection{Shadow explanation}

Brillouin's shadow explanation does not require any explicit consideration of diffraction [4]. Rather, it recognizes that the dark shadow observed immediately behind a large opaque particle must be a consequence of destructive interference between the incident and scattered waves. To see how this explains the paradox, the scattered wave is separated into a reflection wave and a shadowforming wave (SFW), recall Section 2. The SFW achieves cancellation of the incident wave in the near-field shadow zone, whereas the reflection wave is the remaining part of the scattered wave. Now suppose that the opaque particle is nonabsorbent, i.e., $\operatorname{Re}\{m\} \rightarrow \infty$, a reflector. Since the SFW cancels the incident wave, it must carry an energy $I{ }^{\text {inc }} C$ geo along the forward direction. Moreover, because the particle is nonabsorbent, $C^{\mathrm{abs}}=0$. Therefore, Eq. (10) 
shows that the reflection wave must also carry an energy $I^{\text {inc }} C^{\text {geo }}$ (in all directions) in order to compensate for the missing flow in the shadow [31]. Since there is no absorption, the contribution to $C^{\text {ext }}$ from the reflection and SFW are equal, hence $C^{\text {ext }}=2 C^{\text {geo }}$. Examples of work promoting this explanation can be found in [4,6,31] and the quantum mechanical analog in [33].

Conceptually, it is difficult to see how this explanation could apply to small and highly refractive or large and semi-transparent particles. This is because in either case there is not necessarily a well-defined shadow immediately behind the particle. Nevertheless, Lai et al. are able to use the SFW concept to explain the occurrence of the paradox for semi-transparent cylinders with large size parameters [32]. The deeper insight missing from Brillouin's explanation is that the cancellation of the incident wave in the shadow is due to EO cancellation of that wave within the particle. One can see this directly in (e) of Fig. 6 where the EO cancellation wave is generated across $\mathcal{S}^{\text {ill }}$ and travels through the particle into its external near-field zone.

\section{Conclusion}

By expressing a particle's scattered wave in terms of the internal wave, a connection is made as the Ewald-Oseen theorem appears in the formalism describing the extinction cross section. This theorem requires that the incident wave be canceled inside any particle by the particle's own internal wave. The cancellation is achieved by destructive interference from secondary radiation attributed to a source derived from the internal wave. Several illustrations, presented in Figs. 3-6, are used to understand how this source and its secondary radiation are connected to the cross section. Provided that the degree of refraction within the particle is large, as quantified by the phase shift parameter, the cancellation of the incident wave is achieved by the source associated with the particle's geometrically illuminated surface. This same source is also responsible for producing the external scattered wave. Consequently, because of its dual role, the internal-wave-derived source has twice the magnitude expected. This factor of two is then ultimately communicated to the cross section through the external scattered wave. Thus, one sees that it is the Ewald-Oseen theorem's requirement that the incident wave be canceled inside of a particle that explains why the cross section is twice the value expected from geometrical optics. This explanation predicts that $C^{\text {ext }} \rightarrow 2 C^{\text {geo }}$ for small particles provided that they are highly refractive; a fact demonstrated by exact theory but not predicted by the canonical explanations of the paradox.

\section{Acknowledgments}

The authors are thankful for discussions with Gorden Videen, Michael Mishchenko, O. Larry Weaver, Steve Hill, Dave Ligon, Leonid Dombrovsky, Rodolphe Vaillon, and two anonymous reviewers.

\section{References}

[1] Bohren CF, Huffman DR. Absorption and scattering of light by small particles. New York: Wiley; 1983 [Section 4].

[2] Born M, Wold E. Principles of optics. Cambridge: Cambridge University Press; 1999 p. 721-2.

[3] van de Hulst HC. Light scattering by small particles. New York: Dover; 1981 p. 103-8.

[4] Brillouin L. The scattering cross section of spheres for electromagnetic waves. J Appl Phys 1949;20:1110-25.

[5] van de Hulst HC. On the attenuation of plane waves by obstacles of arbitrary size and form. Physics 1949;15:740-6.

[6] Kerker M. The scattering of light and other electromagnetic radiation. New York: Academic; 1969 p. 106-8.

[7] Nussenzveig HM. Diffraction effects in semiclassical scattering. Cambridge: Cambridge; 1992 p. 55.

[8] Newton RG. Scattering theory of waves and particles. New York: Dover; 2002 p. 68

[9] Friedlander SK. Smoke, dust, and haze: fundamentals of aerosol dynamics. New York: Oxford; 2000 p. 130.

[10] Lock A, Yang L. Interference between diffraction and transmission in the Mie extinction efficiency. J Opt Soc Am A 1991;8:1132-4.

[11] Sinclair D. Light scattering by spherical particles. J Opt Soc Am 1947;37:475-80

[12] Bar-Isaac C, Hardy A. Simple derivation of the factor two in the Mie theory. Am J Phys 1975;43:275-6.

[13] Jones AR. Light scattering for particle characterization. Prog Energy Combust Sci 1999;25:1-53.

[14] Sakurai JJ. Modern quantum mechanics. New York: Addison Wesley Longman; 1994 p. 406-10.

[15] Berg MJ, Chakrabarti A, Sorensen CM. General derivation of the total electromagnetic cross section for an arbitrary particle. JQSRT 2009;110:43-50.

[16] Mishchenko MI. Gustav Mie and the fundamental concept of electromagnetic scattering by particles: a perspective. JQSRT 2009;110:1210-22.

[17] Mishchenko MI, Travis LD, Lacis AA. Scattering, absorption, and emission of light by small particles. Cambridge: Cambridge; 2002 [Section 2.8].

[18] Berg MJ, Sorensen CM, Chakrabarti A. Extinction and the optical theorem. Part I. Single particles. J Opt Soc Am A 2008;25:1504-13.

[19] Berg MJ, Sorensen CM, Chakrabarti A. Extinction and the optical theorem. Part II. Multiple particles. J Opt Soc Am A 2008;25: 1514-20.

[20] Mishchenko MI, Berg MJ, Sorensen CM, van der Mee CVM. On definition and measurement of extinction cross section. JQSRT 2009;110:323-7.

[21] Berg MJ. A microphysical model of scattering, absorption, and extinction in electromagnetic theory. PhD dissertation, Kansas State University, Department of Physics, Manhattan; 2008.

[22] Mishchenko MI, Travis LD, Lacis AA. Multiple scattering of light by particles: radiative transfer and coherent backscattering. Cambridge: Cambridge University Press; 2006 [Section 3.1].

[23] Tai CT. Dyadic green functions in electromagnetic theory. New York: IEEE Press; 1994 p. 300.

[24] Tsang L, Kong JA, Ding KH, Ao CO. Scattering of electromagnetic waves: theories and applications. New York: John Wiley \& Sons; 2001 [Chapter 2].

[25] Cruickshank DWJ. P.P. Ewald and his dynamical theory of X-ray scattering. Oxford: Oxford University Press; 1992 [Chapter 12].

[26] Rothwell EJ, Cloud MJ. Electromagnetics. New York: CRC; 2001 p. 67.

[27] Jackson JD. Classical electrodynamics. New York: Wiley; 1999 Section 10.10.

[28] Harrington RF. On scattering by large conducting bodies. IRE Trans Antennas Propag; 1959:150-3.

[29] Harrington RF. Time-harmonic electromagnetic fields. New York: McGraw-Hill; 1961 p. $125-8$.

[30] King RWP, Wu TT. The scattering and diffraction of waves. Cambridge, MA: Harvard University Press; 1959 p. 77-9, 205-13.

[31] Morse PM, Feshbach H. Methods of theoretical physics. New York: McGraw-Hill; 1953 p. 1381.

[32] Lai HM, Wong WY, Wong WH. Extinction paradox and actual power scattered in light beam scattering. J Opt Soc Am A 2004;21: 2324-33.

[33] Keller JB. Quantum mechanical cross section for small wavelengths. Am J Phys 1972;40:1035-6. 